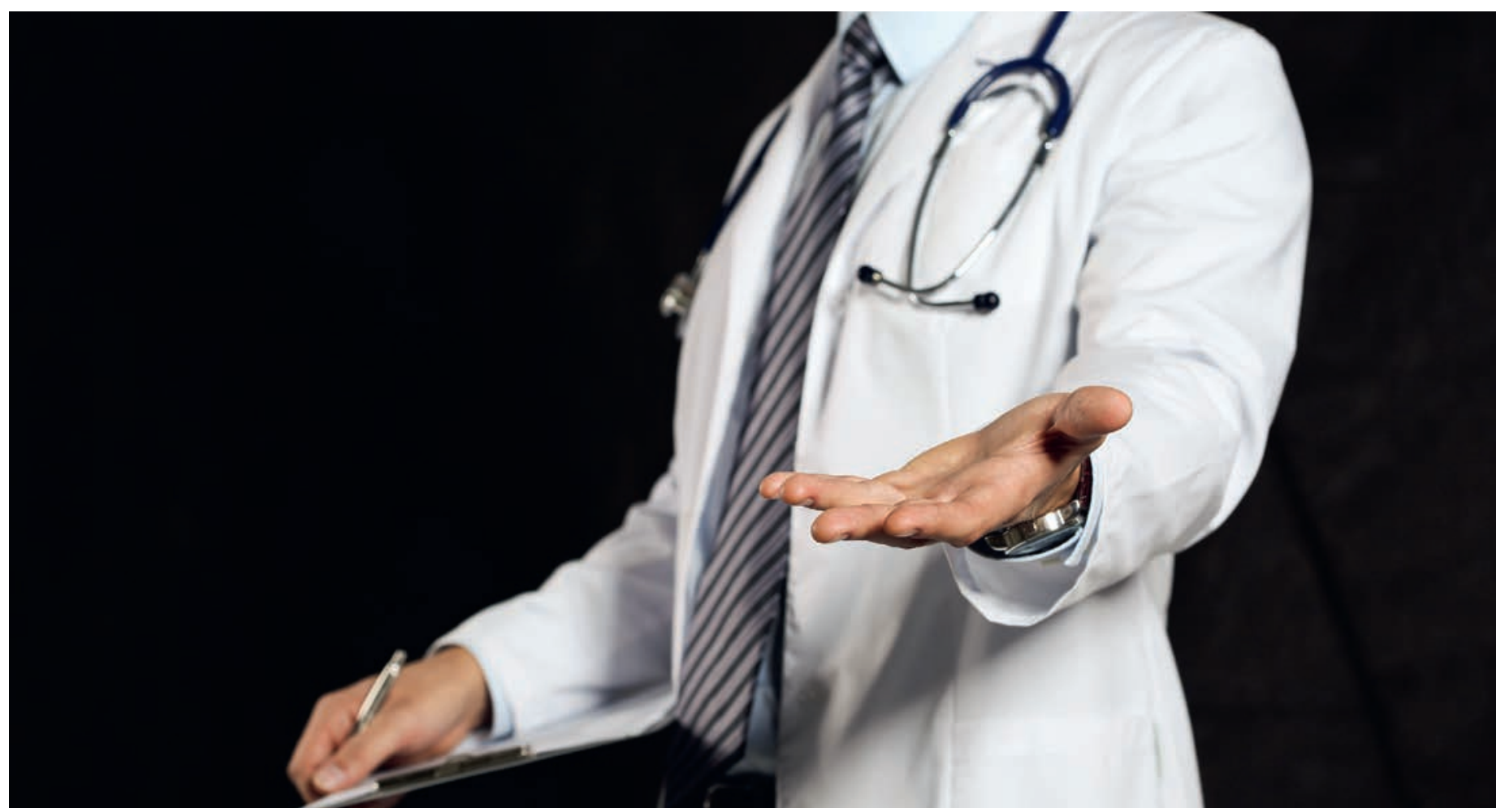

\title{
Kennen Sie die weissen Fremdenführer?
}

\section{Andreas Gosztonyi}

Dr. med., Facharzt für Allgemeine Innere Medizin, Mitglied FMH

Wenn Sie noch nie selber in Leiden waren, ist es gar nicht so schlimm, dass Sie den Ort, bzw. die Gegend, die auf keiner Landkarte zu finden ist, bislang nicht persönlich kennen lernten. Aber vielleicht kennen Sie Menschen aus Ihrem Umfeld, die selber schon dort waren, und haben von deren Erfahrungen gehört. An diesem fremden, ungemütlichen und unangenehmen Ort soll nämlich die Orientierung äusserst schwierig und überfordernd sein. Zum Glück gibt es dort aber ein breites und gutes Angebot an Fremdenführern, die liebend gern ihre Dienste zur Verfügung stellen.

Das Auffällige an diesen Orientierungshelfern ist, dass alle stets in Weiss gekleidet sind. So sind sie schon von weit her für jede Frau und jeden Mann als Helfersfigur erkennbar; ihre helle Erscheinung stellt zudem einen erlösend anmutenden Orientierungspunkt mitten in der dunklen und düsteren Landschaft dar. Bei den ver- schiedenen Reiseberichten der Besucher von Leiden lässt sich eine grosse Divergenz in der vorherrschenden Berufsethik bezüglich der Honorarvorstellungen erkennen. Diese Unterschiede lassen sich, bezogen auf eine Stunde Arbeit, simplifizierend in drei Kategorien einteilen.

In der ersten Gruppe stehen diejenigen, die für eine effektiv geleistete Stunde Arbeit, in der sie einen hilfesuchenden orientierungslosen Menschen zu mehr Wohlbefinden führen, auch wirklich nur ein angemessenes Stundenhonorar für sich beanspruchen. Von der Fachgesellschaft der weissen Helfer gibt es hierfür einen für alle wegweisend angemessenen Normwert. Diese Empfehlung wird im Alltag aber auf mannigfaltigste Art und Weise umgangen und uminterpretiert. So kommen wir zur nächsten Gruppe. Die Vertreter dieser zweiten Gruppe machen die genau gleiche 
Arbeit wie die vorherig Genannten; sie argumentieren aber zusätzlich mit ihren besonderen, ausgefeilten und routinierten Fähigkeiten, die es ihnen ermöglichen, dass sie eine Führung, die früher zwar zugegebenerweise viel Zeit beanspruchte, heute viel schneller, effektiver und dadurch zum Wohle des Betroffenen

\section{Ihre helle Erscheinung stellt einen erlösend} anmutenden Orientierungspunkt mitten in der dunklen und düsteren Landschaft dar.

viel effizienter verrichten können. Aus dieser Mehrleistung schlussfolgern sie, dass sie für ihren wertvollen Dienst von einer Stunde Arbeit nun das Anrecht auf die Bezahlung von zum Beispiel drei Stunden hätten. Das sei der angemessene Preis für ihre Effizienz. Das ist bildlich gesprochen, wie wenn der heutige Taxichauffeur für seine Fahrstrecke den zeitmessenden Taxometer so eichen würde wie damals, als er noch mit der Pferdekutsche unterwegs war, dies aber mit dem Wert der heutigen Minutage multipliziert. Wahrlich eine beeindruckende uneigennützige Effizienz.

$\mathrm{Zu}$ dieser Vorgehensweise gibt es aber noch eine weitere Steigerung. Denn die Exponenten der letzten Gruppe können nicht nur schnell und effektiv die Gäste im Dunkeln führen, sondern sie stellen auch noch eine Veränderung beim Fremden her, und zwar

\section{Von der Fachgesellschaft der weissen Helfer gibt es einen für alle wegweisend angemesse- nen Normwert.}

derart, wie er es so vorher noch nie hatte. So darf er nebst der Orientierungshilfe auch gleich an sich selbst noch einen operierten oder technischen Mehrwert nach der Führung behalten und mit nach Hause nehmen. Die Exponenten dieser Denkweise sind überzeugt, dass eine derart anspruchsvolle Tätigkeit eine Entlohnung rechtfertigt, die ein $\mathrm{x}$-Faches eines «herkömmlichen» Stundenansatzes beträgt. Denn in der Geschichte der Menschheit war es ja bislang immer so, dass nur die wertvollsten Mitglieder einer Gesellschaft das Recht hatten, ein Schwert zu führen. Dies hat sich bis heute auch dann nicht geändert, wenn das sieg- und heilbringende Schwert zu einem Skalpell, einem Strahl oder einem sonstigen technischen Hilfsinstrument reduziert und mutiert ist.
Zusammenfassend lässt sich demnach festhalten, dass in der Gilde der weissen Führer ein von der üblichen Norm abweichendes Verständnis von Zeit, Effizienz und Mehrwert existiert. Da der orientierungslose Hilfesuchende all diesen Machenschaften, Tricks und Effizienzoptimierungen hilflos ausgeliefert ist, bräuchte es externe Regulatoren, die diesem Treiben einen Riegel vorschieben könnten. Dies könnte aber voraussichtlich wiederum andere und neue Probleme mit sich bringen. Darum bleibt seit vielen Jahren alles beim Alten und Bewährten. Dies wird übrigens als «Besitzstandwahrung» bezeichnet.

Sind die Kunden der weissen Fremdenführer wirklich so hilflos und ausgeliefert? Oder könnten sie vielleicht

\section{Könnten die Kunden eine zentrale Kontroll- funktion übernehmen, wenn ihnen mehr Informationen zur Verfügung stünden?}

sogar eine zentrale Kontrollfunktion übernehmen, wenn ihnen mehr Informationen zur Verfügung stünden? Was würde denn passieren, wenn in Zukunft auf den Rechnungen klar und verständlich die effektiv aufgewendete Zeit im Mittelpunkt stehen würde und die Effizienz oder irgendein technischer Mehrwert gesondert und zeitunabhängig aufgeführt wäre? Somit könnte jeder mündige Kunde auf der Rechnung kontrollieren, ob er eine, drei oder $\mathrm{x}$ Stunden bezahlt. Wie gross könnte mit dieser kleinen Veränderung auf jeder einzelnen Rechnung der Sparnutzen für die gesamte Gesellschaft sein? Und wer fühlt sich dazu verantwortlich, eine solche Idee verbindlich umzusetzen? Die weisse Gilde selbst oder die Instanz, die über ihr steht? Ersteres wäre äusserst lobenswert und erfreulich, aber entspricht nicht der Realität. Die Hoffnung bleibt also, dass eine eingreifende Veränderung schneller kom-

In der Geschichte der Menschheit war es immer so, dass nur die wertvollsten Mitglieder einer Gesellschaft das Recht hatten, ein Schwert zu führen.

men wird als der unweigerlich bevorstehende Kollaps. Dies wird aber jene, die im Moment am meisten vom vorherrschenden System profitieren, dannzumal kaum interessieren, denn wie schon bei den alten Kriegern gilt heute immer noch: «Après nous le déluge!»

Bildnachweis

(c) Megaflopp | Dreamstime.com 